\title{
Approche ethnologique des migrations clandestines subsahariennes
}

L'aventure, ou de l'ontogenèse à la conquête de l'honneur

Ethnological Approach of Sub-saharan Irregular Migrations. The Adventure, or from the Ontogenesis to the Conquest of Honour

\section{Matthieu Louis}

\section{CpenEdition}

Journals

Édition électronique

URL : http://journals.openedition.org/etudesafricaines/17412

DOI : 10.4000 /etudesafricaines. 17412

ISSN : $1777-5353$

Éditeur

Éditions de l'EHESS

\section{Édition imprimée}

Date de publication : 16 septembre 2013

Pagination : $547-570$

ISBN : 978-2-7132-2388-4

ISSN : 0008-0055

\section{Référence électronique}

Matthieu Louis, " Approche ethnologique des migrations clandestines subsahariennes », Cahiers d'études africaines [En ligne], 211 | 2013, mis en ligne le 23 octobre 2015, consulté le 02 mai 2019

URL : http://journals.openedition.org/etudesafricaines/17412; DOI : 10.4000/etudesafricaines. 17412 


\title{
Approche ethnologique des migrations clandestines subsahariennes
}

\author{
L'aventure, ou de l'ontogenèse \\ à la conquête de l'honneur
}

\section{Migrations clandestines et anthropologie}

Les migrations clandestines africaines vers l'Europe ne sont pas un phénomène nouveau. Dans les années 1930, poussés par un système colonial coercitif, des clandestins embarquaient dans le port de Dakar en se cachant dans les cales des bateaux pour ensuite débarquer dans les grandes villes portuaires métropolitaines en quête d'emploi (Ndiaye 2008 : 142). Toutefois, la reconnaissance par les États européens du désir d'Occident qui porte certains Subsahariens à braver les périls d'une transhumance spectaculaire et mortifère est, elle, une prise de conscience récente. Les événements de Ceuta et Melilla en septembre $2005^{1}$ et les naufrages en pleine mer des pateras constituent aujourd'hui la vitrine médiatique de ces phénomènes migratoires singuliers, les médiascapes ${ }^{2}$ européens et africains produisant dès lors des scenarii fortement idéologisés à partir des fragments de la réalité vécue par ces aventuriers ${ }^{3}$ contemporains.

1. En septembre 2005, des centaines de Subsahariens munis d'échelles fabriquées artisanalement et à divers endroits, attaquèrent massivement les grillages des enclaves espagnoles de Ceuta et Melilla, îlots européens situés sur le territoire marocain. Plusieurs assaillants trouvèrent la mort pendant ces assauts suite aux réactions brutales des autorités espagnoles et marocaines.

2. La notion de médiascapes est empruntée à Arjun APPADURAI (2005 : 73-74), celuici la définissant comme étant «à la fois la distribution des moyens électroniques de produire et de disséminer de l'information [...] et les images créées par ces médias. [...] Les médiascapes, qu'ils soient produits par des intérêts privés ou étatiques, tendent à être des comptes rendus fondés sur l'image et le récit de fragments de réalité ».

3. Les mots en italique, issus de l'ethnographie, indiquent les termes qu'emploient les aventuriers dans leurs discours. 
« Au moins 50 Africains ont péri en tentant de rejoindre les îles Canaries en bateau depuis le Sénégal, a indiqué mardi la police espagnole, après localisation de l'embarcation qui dérivait depuis plus de quinze jours. C'est l'une des tentatives d'immigration clandestine les plus meurtrières parmi toutes les traversées effectuées par des Africains tentant de fuir la pauvreté en rejoignant la porte d'entrée la plus méridionale de l'Europe $» 4$.

La translation sémantique de la figure du migrant en agent miséreux déterminé par un environnement local réputé exangue s'opère à travers un discours dominant agissant comme «un processus de victimisation des migrants » (Schmitz 2008 : 5). L'information ainsi communiquée au plus grand nombre compose la vision misérabiliste des mouvements migratoires clandestins subsahariens, celle-ci prenant valeur d'interprétation conventionnelle dans les productions discursives de nature politique ou médiatique ${ }^{5}$. En outre, ces travers interprétatifs, connotés socialement et culturellement, se retrouvent également dans le champ scientifique, des travaux universitaires soulignant le caractère victimaire de ces « fuites » migratoires : «Les migrants fuient la misère » (Bredeloup \& Pliez in Alioua $2008: 698$ ) ; « [...] la volonté des jeunes de fuir le continent par désespoir » (Sarr 2008 : 716) ; «[...] les exilés d'Afrique subsaharienne poussés [...] à fuir la misère économique de leur pays pour survivre» (Lépine \& Valluy 2006 : 100). Ainsi, cette chaîne d'idées et de termes, qui caractérise le discours commun et savant, représente-t-elle une doxa imprégnée du schéma issu de l'économie classique et utilitariste de l'homo oeconomicus excluant, d'une part, la dimension imaginaire de l'être humain et, d'autre part, les contextes sociaux et culturels de production des pratiques. Toutefois il ne s'agit pas d'écarter du propos l'argument économique comme élément compréhensif des départs en migration, mais bien de démontrer sa vacuité tant que l'interprétation omet de souligner la dimension symbolique qui régit in fine toute action humaine. L'héritage conceptuel de l'anthropologie économique et le paradigme du don autorisent ainsi à repenser ces phénomènes à la lumière d'une économie qui figure symboliquement les conduites comme des actes sociaux et culturels. Autrement dit, les échanges humains, quelle que soit leur nature (matérielle/immatérielle), n'ont de signification que comme traduction symbolique du social et du culturel. De ce point de vue, les capitaux financiers et les capitaux culturels apparaissent indivisibles des capitaux symboliques, ces derniers déterminant, au tamis de leur importance, l'image et la position que leur propriétaire possède auprès de son entourage. En effet, la logique du don telle qu'elle est observable met en lumière les enjeux symboliques qui structurent la vie sociale dans un environnement où, bien que de plus en plus individualisés, les espaces communautaires demeurent les lieux

4. Immigration clandestine : 50 Africains meurent en tentant de rejoindre les Canaries, Agence Reuters, 6 novembre 2007.

5. Le reporter Serge DanIEL (2008: 17), dans Les routes clandestines, décrit dans ce sens le clandestin comme étant «d'abord quelqu'un qui fuit la pauvreté ». 
anthropologiques privilégiés de l'interaction et de l'imposition du statut social. La lecture strictement matérielle de la pauvreté oblitère les enseignements de l'anthropologie économique qui, par l'entremise de Marshall Sahlins (1976 : 80), affirme que la pauvreté ne signifie pas une faible quantité de biens ou une relation entre moyens et fins mais avant tout une relation d'homme à homme, un statut social. Ainsi, en rejetant la tentation d'une analyse mono-factorielle, est-il certain que l'hypothèse de la pauvreté telle qu'elle est généralement envisagée ne peut être érigée comme explication exclusive de l'existence de ces circulations humaines.

De surcroît, la recherche scientifique actuelle sur les migrations clandestines articule profondément son propos autour des logiques circulatoires à l'œuvre dans ces phénomènes à l'aide d'un ancrage théorique insufflé par les sciences géographiques. Le cadre conceptuel comprend un travail descriptif élaboré à partir d'une « perspective territoriale » d'où émergent les notions d' « espace », de « territoire », de «frontière », de « mobilité », de « circulation » ou de « réseau ». En d'autres termes, le paradigme migratoire apparaît généralement comme un dialogue entre le mouvement et l'espace, entre la mobilité et la territorialité. Un ouvrage récent de G. Cortès et L. Faret (2009) re-questionne ainsi les phénomènes migratoires contemporains en adoptant une approche centrée autour de l'idée de «circulation migratoire », un concept qui « correspond à une démarche de problématisation des phénomènes de mobilité physique des hommes, avec leur itinéraire, les pratiques effectives et affectives des espaces parcourus ${ }^{6}$ (Hily 2009 : 23-24). Ces études revendiquent l'apport de la tradition anthropologique pour deux raisons. La première est d'ordre méthodologique dans la mesure où les chercheurs conduisent des enquêtes ethnographiques de terrain. La seconde est de nature interprétative car leurs démonstrations suggèrent que la démarche anthropologique est «à même de comprendre les chaînes temporelles, les interactions et les négociations dans lesquelles les circulants transnationaux évoluent» (Cortès \& Faret 2009: 18). Il s'agit alors pour ces auteurs d'étudier, dans une certaine mesure, les sociabilités et leurs multiples organisations mises en rapport avec les territoires de la circulation transnationale.

Néanmoins, il demeure légitime de s'interroger sur la place réelle accordée à la pratique ethnologique — à savoir l'interprétation savante des éléments empiriques recueillis sur le terrain — dans le cadre des recherches sur les migrations clandestines. À la lecture de nombreux travaux existant dans ce domaine, l'anthropologie apparaît comme marquée du sceau de la

6. Selon l'auteure, M.-A. HILY (2009 : 24) cette notion, accompagnée des concepts de territoires circulatoires, de réseaux transnationaux et de diasporas, se différencie de celle de migration, et «conduit à réinterpréter le "champ d'expérience" et l' "horizon d'attentes" des migrants et permet de reposer à nouveaux frais la question des temporalités sociales, des rapports et des hommes à l'espace et à leur histoire identitaire ». 
périphéricité, s'insinuant ici et là dans les anfractuosités des champs épistémologiques qui lui sont connexes. Autrement dit, invoquée régulièrement dans des approches disciplinaires d'horizons variés, elle n'acquiert que rarement le statut d'épistémè à part entière. Or l'ethnologie constitue historiquement un projet scientifique qui se définit autour d'un ensemble de paradigmes, de méthodes et d'outils d'analyse distincts. L'ethnologie, pensée comme entreprise de traduction culturelle des conduites humaines (Kilani 2009: 81), impose de replacer l'analyse de ces dernières au sein des schèmes culturels et sociaux qui président à leur élaboration. Aussi, appliquée aux migrations clandestines subsahariennes, s'agit-il pour nous de contextualiser ces phénomènes par l'exploration des systèmes sémantiques de référence qui guident les stratégies des aventuriers. Les travaux des géographes mettent en évidence des mondes «en train de se faire» au gré des parcours de mobilité (Hily 2009 : 26) où les enjeux circulatoires s'actualisent sans cesse mais ont, en revanche, le travers d'évacuer le symbolique de l'action humaine, conçu comme structure efficace mobilisant les individus et les groupes autour d'une idée ou d'une action (Kilani 2009: 108). Par conséquent, si «ce qui intéresse le géographe est que l'interaction sociale est spatialement située » (Schaeffer 2009 : 70), pour l'ethnologue ce qui importe est d'interpréter les pratiques à travers les univers structurants de significations dans lesquelles elles prennent corps.

L'approche analytique de ce travail repose sur la constatation, par le décentrement du regard qu'impose la pratique ethnologique, que des acteurs des migrations clandestines ouest-africaines s'attribuent l'identité générique d' « aventurier ». En effet, quatre ethnographies conduites au Burkina Faso entre 2008 et 2010 mettent en évidence l'inexistence dans les discours du terme «migrant », systématiquement substitué à celui d' " aventurier ». Être aventurier, c'est revendiquer positivement le sentiment d'appartenir à une communauté imaginée hétérogène aux contours flous qui participe aux importantes circulations humaines en Afrique de l'Ouest ${ }^{7}$. Autrement dit, est aventurier celui qui se prétend aventurier. Dès lors, il est à exclure de l'interprétation qui suit tout migrant ne se reconnaissant pas en tant que tel ${ }^{8}$. Souvent intégré sous le vocable de «migrant économique», «travailleur migrant » ou «target worker », et plus récemment celui de «transmigrant », l'aventurier, en tant que catégorie subjective d'analyse, demeure toutefois inclassable dans la taxinomie classique du paradigme migratoire. Questionner la figure de l'«aventurier», et non celle du «migrant» et ses diverses

7. Toutefois, il est à souligner que l'«aventurier» est une notion partagée dans d'autres régions africaines, des entretiens menés auprès de ressortissants camerounais et tchadiens tendent à valider cette hypothèse.

8. Des chercheurs comme Mahamet Timera ou Anaïk Pian notent par exemple que certains transmigrants présents au Maroc rejettent ce statut particulier d'aventuriers tels les petits vendeurs de rue sénégalais de Casablanca (PIAN 2009: 13) ou des individus provenant de vagues migratoires plus anciennes (TIMERA 2009: 185-186). 
déclinaisons, nous projette dans un univers polysémique que cette dernière peine à dévoiler. Par conséquent, sonder le point de vue de l'acteur met à jour des expériences et des modes opératoires singuliers de la mobilité, un imaginaire du voyage et un ethos qui puisent leur sens à la confluence des référents sociaux et culturels originels.

Ce texte présente ainsi une description modale construite autour d'éléments subjectifs communs à une vingtaine de récits de vie d'aventuriers et relayée par trois trajectoires subjectives considérées comme typiques qui constituent le socle de mes interprétations ${ }^{9}$. En fondant l'approche sur l'étude de la trame sémiologique interne, l'analyse des représentations émiques autorise à tracer l'esquisse d'une acception ontologique, sociale et culturelle de l'aventure telle qu'elle est perçue par ses acteurs.

\section{Éléments définitoires de l'aventure}

Apparaissant comme un truisme parce qu'étant le principe même de l'aventure, le premier élément de définition se cristallise autour de la notion de «mouvement». Faire l'aventure, c'est sortir, c'est quitter les siens, c'est partir de chez soi. L'aventurier prend la route ou le chemin, il s'exile en traversant des régions inconnues. En empruntant la voie, il bouge de droite à gauche («le ting-tang »), il avance au gré des événements, vers la destination choisie, selon son imagination, selon sa faim ${ }^{10}$. Dès lors, l'aventurier devient un sujet délocalisé, extrait de ses cercles de socialité primaire, qui circule entre des espaces temporels de fixation spatiale plus ou moins longs. À ce titre, l'aventure revêt différentes formes de circulations territoriales et n'est pas exclusivement transnationale. Autrement dit, si les migrations clandestines mettent incontestablement les attributs de l'aventure, les migrations internes (rurales-rurales, rurales-urbaines, urbainesrurales) n'en sont pas moins des aventures. En effet, quitter sa région natale pour rallier la capitale, sortir de son village pour aller travailler dans des champs établis dans une région agricole source d'emplois rémunérateurs sont, par exemple, des conduites également considérées comme des aventures par les interlocuteurs.

Ainsi l'exode rural, la migration des Haalpulaar vers les bassins miniers du Zaïre dans les années 1950-1960 (Bredeloup 1994), la migration coloniale du navétanat (Amselle 1976), les migrations burkinabè vers les champs

9. Adepte d'une démarche inductive « à l'aveugle », j'ai conduit mes recherches à Ouagadougou et Bobo-Dioulasso ainsi que dans les villages de Yamtenga, Salagi (moose) et Nabou (gurunsi). Au-delà des innombrables discussions informelles auprès de mes interlocuteurs privilégiés et du tout-venant s'ajoute une trentaine d'entretiens semi-directifs enregistrés dont plus d'une vingtaine permet de tracer les trajectoires biographiques d'acteurs se proclamant «aventuriers».

10. Il faut comprendre ce dernier terme comme un synonyme d' « envie », de « désir» et non comme une expression relative au besoin physique de se nourrir. 
de cacao ivoiriens et ghanéens, les flux contemporains dirigés vers des pôles africains économiquement attractifs (Gabon, Afrique du Sud, Guinée équatoriale, Libye, etc.) ou vers l'Europe sont, entre autres, des logiques migratoires qui s'inscrivent dans le mode mobilitaire de l'aventure. Celle-ci se perçoit donc d'abord à travers une approche multiscalaire que le parcours individuel de Lamine synthétisé ici exprime typiquement:

« J'ai quitté chez moi à pas d'âge. À quinze ans, je suis parti pour la Côte-d'Ivoire. Je n'ai averti personne de la famille. J'ai vendu quelques affaires et je suis monté dans le train. Des amis m'ont accompagné à la gare pour voir si j'osais m'en aller. Je ne me suis pas découragé et j'ai sauté dans un wagon quand le train démarrait. Nous avions tous la Côte-d'Ivoire en tête, c'était l'endroit où tout se passait. Au cours de mon aventure, j'ai été accueilli par plusieurs familles qui m'ont pris en pitié, je n'étais encore qu'un enfant. Puis une tante m'a retrouvé et m'a hébergé à Abidjan. Mais elle a fini par apprendre que j'étais parti de chez moi sans la bénédiction de mes parents. Elle m'a renvoyé à Bobo par le train cinq mois après mon départ.

Ma famille était contente de me revoir et de me savoir en bonne santé. Mais ce que j'avais vu était désormais une obsession. La vie là-bas était différente et m'attirait comme un aimant. Quelques mois se sont écoulés avant que je ne reparte pour Abidjan avec un ami. Ce n'était pas une question d'argent, je vivais dans une famille aisée et respectée : mon père est un marabout très connu et il dirige une école coranique réputée. C'était en 1986 et dès lors j'ai vécu dans la capitale ivoirienne jusqu'en 1994. Je suis revenu plusieurs fois pour visiter ma famille, pour demeurer proche de mes parents mais ma vie était à Abidjan. J'y ai appris la soudure et je gagnais bien et facilement ma vie. Je vivais ma jeunesse sans entraves entouré d'amis.

Puis la crise a frappé le pays, le climat social était tendu, surtout pour les étrangers. La vie n'était plus aussi simple et l'envie de découvertes s'insinuait dans nos esprits, à moi et un ami ivoirien de Yopougon. On planifiait l'aventure au Gabon par la voie terrestre. On était ambitieux, on visait de grandes choses. Malheureusement on a échoué à Lagos, au Nigeria. Cette ville est comme un puits, une fois tombé dedans, il faut construire l'échelle soi-même pour s'en sortir. J'y ai vécu les pires moments de ma vie, j'ai expérimenté la faim, la misère, l'injustice, l'égoïsme et la perfidie des hommes, la solitude, et j'ai vu la mort. Toute cette souffrance juste pour survivre dans cette ville hantée [...] On a abandonné notre projet et, après deux années de galère, on a rallié le Ghana pour s'installer à Accra, en 1996.

J'y ai habité six ans. Je travaillais dans l'artisanat et faisais du commerce. Dans l'intervalle, je suis retourné au Nigeria pour retenter ma chance, mais sans succès. Je suis revenu à Accra sans avoir pu trouver la voie. Mais à force de travail et de persévérance, je me suis constitué une petite économie. Pour la première fois de ma vie j'ai loué une maison pour moi seul ! Je me suis constitué un stock de marchandises car je désirais faire du commerce itinérant, travailler tout en voyageant. Je suis donc parti à Ouagadougou en 2001 pour commercer. Je n'imaginais pas à ce moment que je ne reviendrais pas à Accra. Mon affaire a échoué et je me suis trouvé bloqué à Ouagadougou.

J'ai accepté cette situation parce que j'étais au Faso. À 31 ans, après avoir passé la moitié de ma vie à l'extérieur, j'ai pris conscience que mon propre pays m'était étranger. La pire des souffrances a aussi disparu à mon retour. Quand on est à l'extérieur, on se sent prisonnier de soi parce que l'on vit avec un fardeau. Ce fardeau, c'est la prudence. Tu dois tout contrôler et te maîtriser, cela épuise ton énergie au quotidien. Tu vis dans l'angoisse et la peur. Tu souffres à l'intérieur de toi car tu sais que les problèmes que tu affrontes dehors n'existent pas en famille. 
En aventure, tu te sens orphelin. Avec de la chance tu rencontreras des gens qui connaissent la pitié mais rien n'est sûr. Si tu sors à l'aventure, il ne faut pas te perdre en route, il faut connaître le chemin de la maison.

Cependant, même si je m'étais rapproché de la famille, j'étais toujours à l'aventure, l'aventure à la capitale, celle de mon pays. J'ai eu de nouveaux projets. Je suis devenu guitariste professionnel et j'avais des contrats pour jouer dans des maquis et des studios d'enregistrement. En 2005, je suis parti à Bamako pour vendre des habits dans l'espoir d'amasser assez d'argent pour produire un disque. Malheureusement cela ne s'est pas achevé comme prévu. Je me suis retrouvé sans fonds dans la capitale malienne et j'ai dû me débrouiller au jour le jour. J'avais un petit frère boutiquier sur un marché de Bamako. Je suis allé le voir et il m'a donné l'argent pour que je puisse rentrer au Burkina. Jusqu'à aujourd'hui, je vis au pays, entre chez moi, à Bobo-Dioulasso, et Ouagadougou. Si je dois repartir à l'aventure, c'est l'Europe, mais légalement car je suis fatigué... » (Lamine, 40 ans, Dioula, BoboDioulasso, décembre 2010).

Au-delà du déplacement territorial, l'aventure se comprend à l'aune des difficultés que comporte une telle entreprise. Elle mobilise des valeurs telles que le courage et l'abnégation, l'oubli de soi-même. Prendre la route, c'est risquer, c'est oser. Le courage, référent axiologique central, est la valeurforce au fondement du défi lancé au destin. L'adage moaga «celui qui a toujours peur n'a pas à manger » répond à l'aventurier qui tente sa chance en s'engageant dans un combat face à la souffrance, une lutte pugnace contre lui-même. C'est la conception d'une destinée écrite à l'avance mais dont on ne connaît pas le déroulement qui imprègne les esprits. En somme, sortir, c'est consulter le dictionnaire de sa vie. Combattre par l'aventure revient à chercher sa vie, à la gagner ou à la vaincre. Aussi, selon les dangers encourus, l'aventure se dévoile comme un acte héroïque : plus les risques pris se rapprochent de la frontière invisible qui sépare la vie de la mort, plus l'intrépidité de l'individu se mue en héroïsme. Le parangon de l'acte héroïque prend véritablement sa forme dans les déplacements au caractère hautement mortifère comme les traversées maritimes ou celles du Sahara. La mort se dessine sur toutes les lèvres, derrière chaque vague, derrière chaque dune, derrière chaque représentant armé des autorités nationales. C'est pourquoi, en vertu des obstacles à surmonter, la migration clandestine vers le Vieux Continent représente aux yeux des aventuriers « la grande aventure ».

\section{"Il n'y a personne qui ne souffre pas dans la vie. »} Jeune moaga, aspirant à l'aventure

Corrélative aux risques, la souffrance s'installe en toile de fond des parcours sinueux de l'aventurier. Elle est synonyme de privations, de maladies, d'humiliations ou d'exactions qui irriguent le vécu des aventuriers. Souvent physiques, elles sont par extension symboliques car elles blessent le moi individuel. La déchirure n'est pas uniquement une expérience corporelle, 
elle est aussi psychologique, elle plonge l'être dans une souffrance qui le vide de sa substance identitaire, qui ronge son idéal. Par conséquent, l'aventure se déploie comme pratique anxiogène où la renonciation à l'identité par le migrant, au départ volontaire, est amplifiée par les actes de déshumanisation ${ }^{11}$ subis lors de son odyssée. Échoué (stranded) à plusieurs reprises, le désœuvrement et l'inactivité accentuent l'anxiété d'un immobilisme dû au défaut de capitaux nécessaires à la poursuite du voyage. Ne pas avoir d'activité rémunératrice est vécue comme une malédiction, car c'est justement grâce à elle que l'individu peut parvenir à atténuer ses souffrances. Le travail marche avec le bonheur et même s'il est lui-même source de souffrance, il cristallise en son sein l'espoir de s'immiscer hors du cercle vicieux de la douleur.

Néanmoins, la souffrance n'est pas exclusive à la pratique de l'aventure. Exprimé différemment, partir en aventure c'est se mettre dans la souffrance, se dresser face au risque, c'est vivre dangereusement, mais la vie quotidienne au sein des contextes sociaux originels arbore, dans une certaine mesure, des potentialités de souffrance similaires. L'éventuelle urgence quotidienne à assumer les besoins primaires, le spectre du chômage, de la maladie, de la mort ainsi que l'angoisse du lendemain peuvent, par exemple, ancrer l'acteur dans une temporalité fixe qui anéantit ainsi toute volonté à se projeter dans l'avenir.

Aussi, comme le souligne Georg Simmel (2002 : 71), « lorsque des deux choses vécues, dont les contenus ne sont guère différents, l'une est éprouvée comme étant une "aventure", tandis que l'autre ne l'est pas, c'est dans la différence du rapport vis-à-vis de la totalité de la vie qu'il faut en chercher la cause ». Cette différence émerge clairement dès que l'on définit l'aventure comme un "mouvement dans l'inconnu ». Ici se situe effectivement le fondement de cette conduite : celui qui se jette dans l'aventure est celui qui brave l'inconnu. Il perd ses repères en s'isolant de ses réseaux d'appartenance sociale. Loin de chez lui, l'aventurier ne peut compter sur les siens. Dépouillé en propre de son individualité, il évolue dans l'inconnu; il est un inconnu, un étranger qui se trouve mis hors du nomos, hors de la loi ordinaire des humains. Son existence se fonde sur la perte d'un lieu, auquel étaient attachés des attributs d'identité, de relation et de mémoire, et sur l'absence d'une nouvelle place sociale ${ }^{12}$ (Agier 2002 : 55). Forcé de se créer des circuits d'interconnaissance nouveaux, l'aventurier improvise, est sur la

11. Nous empruntons cette formule à Michel AGIER (2002: 32). Ces actes prennent la forme de l'oppression policière et militaire, du racket, du racisme et de la xénophobie ou encore de l'ostracisme communautaire. Les conditions de vie des migrants clandestins et les dangers auxquels ils doivent faire face sont finement décrits et rapportés de manière édifiante par plusieurs ouvrages, LÉPINE \& VALLUY (2006), Migreurop (2006), DANiel (2008), GATTi (2008).

12. Nous reprenons à notre compte l'analyse que fait Michel Agier à propos des réfugiés car nous pensons qu'elle est tout à fait applicable aux situations vécues par les aventuriers. 
brèche, ses sens sont sans cesse sollicités. Ainsi, l'aventure implique un déficit de solidarité et de soutien moral qui exacerbe les souffrances relatives à sa mise en œuvre. Souffrir dans le cadre de l'aventure devient une expérience profondément individuelle qui mobilise toutes les ressources personnelles de l'aventurier. L'aventure évalue sa capacité d'adaptation et de réaction à des environnements qui, parce qu'inconnus et non maîtrisés, lui sont hostiles. Par ailleurs, nous retrouvons l'«inconnu» comme élément distinctif de l'aventure dans les référents culturels originels. L'aventurier chez les Moose est pa-weoogo (plur. pa-weto), littéralement «celui qui est resté en brousse». L'aventure signifie aussi l'action de «(re)chercher en brousse » («n bao weoogo »). Pour les Dioula, l'aventure se dit «tungan », l'acte de «chauffer (gan) la brousse/la forêt (tú) » ${ }^{13}$. La brousse comme signifiant culturel symbolise alors cet espace indistinct, non anthropisé en rapport avec le sauvage, l'étrange et l'inconnu ${ }^{14}$.

Dès lors, l'acception émique de l'aventure met particulièrement en lumière ce mouvement dans l'inconnu qui est rupture avec le cours habituel de la vie. L'individu se délocalise dans un univers hétéronome situé « aux bords du monde » (Agier 2002) où rien ne va de soi, où tout reste à faire.

\section{L'aventure ou la fabrique de l'être}

L'aventure est une plongée dans cet inconnu synonyme de limen, espace intermédiaire hors du temps social de référence qui constitue le stade central des processus initiatiques ${ }^{15}$. Dans son acception culturelle, l'initiation se conçoit comme une épreuve dont les principes reposent sur l'accès à de nouvelles connaissances sur le monde qui participent au renouvellement et à la construction de l'être. Elle donne généralement à penser l'ordre de la vie et du monde. Par les connaissances acquises, l'individu envisage différemment l'univers mais il se trouve également changé, dans son Moi le plus profond, il devient autre en aboutissant à la connaissance de lui-même. En d'autres termes, la connaissance est la moelle du processus de maturation sociale généré à travers les pratiques initiatiques ; elle seule permet la rénovation de l'être et sa transformation en un individu socialement pubère. Il

13. Traduction subjective réalisée par Lamine, aventurier dont le récit de vie a été présenté plus haut.

14. Mahamet TimERA (2009: 179) souligne cette même analogie dans la langue soninké avec les termes tere (marcher) et ro gunne (entrer dans la brousse ou la forêt). Il cite également le terme wolof $d u g$ al qui recouvre la même acception (entrer dans la forêt).

15. Le rite d'initiation se comprend à travers la distinction classique en trois phases successives héritée des travaux d'Arnold van Gennep (BELMONT 1981: 24) : la séparation, la marge, l'agrégation. 
se sépare de son état d'enfant — la mort — pour s'agréger au monde des adultes - la renaissance - après une période liminaire qui se charge d'effacer son ancienne identité par l'instruction initiatique.

\section{"Il faut sortir pour connaître. »}

Chef de village (Tengnaaba), ancien aventurier

Au moment de l'analyse des discours des aventuriers, il a été saisissant de constater que le sens de l'aventure se fond sémantiquement dans les représentations de l'apprentissage initiatique issues des cadres conceptuels locaux de référence. L'aventure est une connaissance, un savoir qui permet de comprendre la vie. Quand on prend la voie, on change indubitablement, c'est une métamorphose qui symbolise le commencement de sa vie, une reprise à zéro dont la force motrice est l'espoir de devenir quelqu'un. Généralement, ne pas s'initier revient à «perdre sa vie » (Fiéloux \& Kambou 1993 : 84). Dans le même temps, l'aventurier demeure celui qui part ailleurs, porté par le désir irrépressible de chercher et gagner sa vie. Connaître devient un véritable leitmotiv pour lui. Il faut sortir pour voir la réalité, comprendre le monde, donc enrichir son savoir sur celui-ci.

L'aventure est également un projet ontologique qui se produit à l'aune de l'expérience authentique de la souffrance. Le sens donné à l'aventure se cristallise dans cette propension à affronter la souffrance, celle-ci étant bien souvent élevée au rang de valeur positive : «Il faut souffrir pour réussir » apparaît comme une expression récurrente dans les discours. La souffrance constitue un passage obligé pour atteindre ses objectifs. Les efforts consentis, la dépréciation de l'intériorité de l'individu, les risques encourus, sont autant de points d'accès au succès de l'entreprise humaine. Si le mouvement spatial est « passage matériel ${ }^{16}$, la souffrance, elle, est une épreuve ambivalente. Elle est non seulement source de savoir mais elle est aussi sable mouvant, situation complexe et douloureuse souvent inextinguible. Autrement dit, la souffrance est à la fois pathologie et remède. Elle marque au fer rouge l'expérience pratique de la connaissance dans l'âme de l'aventurier. Celui-ci s'éveille au fil de son itinéraire, apprend, voit et enregistre, découvre de nouvelles données sur le monde. Il se compare aux autres, l'aventure devient le révélateur de sa propre identité, son caractère se modifie, elle n'est autre qu'une expérience initiatique qui ouvre le ritualisant à la connaissance de soi, donc à la maîtrise de son être. L'individu

16. Arnold van Gennep insistait sur la similitude des rites de passage et des passages matériels. Il entendait par passage matériel, un col de montagne, un cours d'eau, le seuil d'une maison ou d'un temple, un portique, la frontière entre deux territoires ou pays, etc. (BELMONT 1981: 23-24). 
se construit au cours de son voyage, telle l'initiation traditionnelle, l'aventure est anthropopoeisis ${ }^{17}$.

Le récit de vie de Bakari exprime cette maturation de l'individu qui s'opère à travers son cheminement initiatique, ce dernier ayant pour conséquence paradoxale de produire un hiatus entre lui et sa famille tout en renforçant l'intégration de Bakari en son sein :

« Je suis issu d'une grande famille. Mon père avait quatre épouses et je suis le fils aîné de sa dernière femme. Mes grands frères ont hérité de l'autorité familiale à sa mort. J'avais alors 19 ans et j'ai dû arrêter mes études car ils ne voulaient plus les financer. Je suis rentré au village et j'ai cherché un travail. Je me suis gravement blessé à la jambe sur un chantier et je ne pouvais plus faire de travaux physiques. J'ai travaillé dans des associations, auprès de la mairie et de l'administration de ma province, le Boulgou. J'ai soutenu des actions de santé publique comme des campagnes de vaccination contre la poliomyélite ainsi que des projets de développement agricole initiés par des ONG.

Cependant je n'avais pas d'avenir au village, je n'arrivais pas à me contenter de cette vie. Puis on m'a accusé injustement de vol et j'ai eu une histoire avec une fille qui est tombée enceinte par ma faute. Sans qu'on puisse l'affirmer, elle était une parente éloignée de ma mère et notre relation a été perçue comme incestueuse. Je me suis senti sali et, malgré mon caractère, je n'ai pas eu le courage de me défendre. Une nuit, j'ai pris mon sac et je suis parti à l'aventure.

Je partais pour le Gabon, mais à 25 ans, je n'étais encore qu'un enfant naïf. Des passeurs m'ont escroqué tout mon argent au Bénin. Je suis rentré au village un temps. La honte persistait et l'aventure demeurait en moi. À la fin de l'année 2001, j'ai vendu mes bœufs puis je suis parti vers la Mauritanie avec 400000 FCFA. À ce moment j'avais l'Espagne en tête et je voulais y arriver quel que soit le moyen. Je suis monté dans un bateau marchand au large de Nouadhibou grâce à des passeurs que j'avais payés. J'étais avec d'autres aventuriers : des Guinéens, des Sénégalais et des Maliens principalement. Nous étions cachés dans un container mais l'équipage nous a surpris et nous a livrés à la police. Dès lors, j'ai abandonné l'idée de l'Europe. Je suis entré au Sénégal et j'ai entendu parler d'opportunités en Gambie. Je m'y suis installé, à Gambisara, un village marka.

Là-bas, je suis vite devenu chef de chantier. J'ai travaillé pendant deux ans et j'ai gagné beaucoup d'argent. Je l'ai investi dans un commerce à Madina Samako. Malheureusement mon affaire a périclité parce que je me faisais racketter par l'armée et la police. J'étais étranger et on me soupçonnait d'être un espion au service des rebelles casamançais qui sévissaient à la frontière. Avant d'être ruiné, je suis entré au Sénégal où j'ai travaillé plusieurs mois comme boy dans des familles aisées de Dakar. Finalement je suis revenu chez moi à la fin de l'année 2006. J'avais presque ma mise de départ en poche.

Je suis rentré au village par la grande porte parce que j'ai eu le courage de m'asseoir avec ma famille pour lui expliquer que j'étais contre tout ce qui m'avait entouré avant que je ne quitte le village, le vol présumé ou l'accusation d'inceste. Nous n'en avions jamais discuté auparavant. Tout le monde m'a écouté et ils ont accepté mes paroles. L'aventure m'a permis de ne plus avoir peur d'eux. Dans un premier temps, c'était l'enfance, je le reconnais. Mais au fur et à mesure de mon aventure,

17. Pour C. Calame et M. Kilani (1999: 7), l'anthropopoeisis, « la fabrication de l'humain », souligne l'idée de «faire », de « construire », de «fabriquer » des êtres humains, ou plus précisément des modèles d'êtres humains. 
j'ai grandi et j'ai commencé à penser que je pouvais affronter mes aînés. On acceptait tout ce que nos grands frères disaient, mais arrivés à un certain point, pourquoi ne pas nous imposer sur certaines choses ? Dès le départ c'était difficile car les jeunes n'étaient pas écoutés. Et c'est l'aventure qui m'a permis d'être entendu parce que j'ai eu la chance de diriger des gens plus âgés que moi en Gambie. Mes ouvriers m'écoutaient et m'acceptaient, ils me respectaient en suivant mes consignes. Alors j'ai compris que j'étais capable de faire face à ma famille.

Aujourd'hui, j'ai ma place au conseil de famille. On m'écoute parce que je connais autre chose. J'ai des idées que l'aventure m'a inspirées et qui peuvent apporter des solutions aux problèmes que rencontre la famille. Peut-être que je n'ai pas d'argent pour la soutenir mais j'ai une vision des choses et une instruction qui peuvent lui être utiles. Et même si mes aînés s'opposent toujours à ce que je veux devenir, on me respecte pour ça » (Bakari, 36 ans, Bisa, Ouagadougou, mars 2009).

Dès lors, nous pouvons parler «d'une traversée initiatique illuminée par l'espoir d'exister en tant qu'être humain » (Boni 2008 : 682). La naïveté, avatar de l'enfance, se dérobe au profit d'une meilleure compréhension du monde. L'individu devient socialement mature, il entame sa mue vers l'âge adulte. Sortir devient donc une quête de re-naissance, une aurore sociale à partir de laquelle un nouvel être voit le jour. L'individu cherche sans relâche à se produire lui-même, c'est pourquoi il est potentiellement toujours un être en devenir. Seule la connaissance acquise lors de l'odyssée autorise cette mutation. Prendre la route, «c'est commencer ta vie, c'est comme quelqu'un qui vient de naître », affirme un ancien aventurier moaga, or, naître au monde, c'est s'agréger dans un espace sans repères, dont on ne maîtrise ni les tenants ni les aboutissants. Aussi, s'engager dans l'inconnu, c'est être comme le nouveau-né, dans son acception traditionnelle, c'est-àdire un «étranger», un saana diraient les Moose.

L'aventure est l'arène du développement personnel de l'individu en quête de savoir et de sagesse. Cette marge — l'aventure — est physiquement un autre monde où l'individu agit hors de sa propre existence historique, « le lieu de toutes les potentialités » (Goguel d'Allondans 2002 : 65), les pires comme les meilleures. Par ailleurs, éloigné de ses cercles sociaux d'interactions privilégiées, l'individu se crée ses propres réseaux d'échanges en s'agrégeant à des communautés imaginées de nature hétérogène ${ }^{18}$. Il y perd souvent son nom, enfoui dans l'ombre de son passé, pour se draper du statut générique de l' « aventurier », du «clandestin », de l' «étranger» ou, exprimé différemment, du non-familier.

Après l'expérience initiatique, le sujet quitte enfin la marge pour réintégrer le monde connu, mais il est autre. De ce point de vue, l'aventure est vécue comme un rite de passage médiatisé par l'accès à des connaissances d'un genre nouveau: d'une part, elles concentrent un savoir-être sur le monde, témoin de la maturation de l'acteur au fil de son parcours. D'autre

18. Certains auteurs parlent de «communautés d'itinérance » (ESCOFFIER $2008: 190)$, de «communautés de migrants » (BRACHET 2009: 115), de «diasporas » ou de «communautés transnationales » (BRUNEAU 2009: 30). 
part, elles sont un savoir-faire pratique, concret, une accumulation de compétences inédites concrétisées par l'incorporation de nouveaux schèmes de pensée, l'apprentissage d'un métier ou la maîtrise de langues étrangères.

En outre, il est essentiel de souligner le désir émis par l'aspirant à l'aventure de s'inventer un avenir hors des structures sociales gérontocratiques. Par le savoir dont il s'instruit loin de chez lui, l'aventurier remet en cause l'harmonie sociale telle qu'elle est établie dans sa communauté d'origine. De son expérience originale, il retient le goût de l'autonomie, de l'indépendance vis-à-vis de l'autorité des aînés, il veut s'affranchir d'un carcan social qu'il juge trop étroit pour devenir quelqu'un. De ce fait, l'aventure, par la transformation ontologique qu'elle implique, s'affiche comme un processus d'individuation ou de différenciation qui éloigne le «nomade » du « sédentaire ». Dès lors, un fossé se creuse entre celui qui est resté, qui s'est mis en arrière et celui qui est sorti pour chercher sa vie ailleurs. Pensée ainsi, l'aventure s'interprète comme un hymne à la liberté, un acte revendicatif du droit à choisir son destin, une tentative de s'affranchir pendant un temps des contraintes inhérentes à l'entre-soi familial en s'aguerrissant au contact d'un monde imaginé mais inconnu en pratique.

"C'est une coutume. [...] II faut que tu sortes d'abord en aventure avant de venir t'installer."

Aspirant moaga à l'aventure

Si, à l'instar de Denis Jeffrey (2005: 36), nous pensons que «ce qui se transmet, d'une génération à une autre, c'est moins la forme d'un rituel que la valeur symbolique qu'il représente », alors nous postulons que les références discursives des aventuriers procèdent des conceptions éducatives énoncées par les cultures locales d'origine et qui sont antérieures au phénomène actuel de l'aventure. Selon Joseph Issoufou Conombo (1989 : 92-95), l'aventure était une pratique sociale instituée dans la société moaga précoloniale. Elle était à certains égards une obligation traditionnelle qui incitait l'adolescent à mobiliser les valeurs de courage, de maîtrise de soi et de perspicacité. Connu sous le nom de « grand voyage », ce passage à la puberté sociale configurait déjà la conception contemporaine de l'aventure. Ces déplacements vers les régions voisines du Burkina Faso actuel conçus comme des épreuves visaient à l'incorporation par les jeunes aventuriers des modèles axiologiques qui concourent à la naissance de l'homme moaga (ibid. : 95). Aussi, cette pratique serait-elle à l'origine des diasporas moose peuplant les pays mitoyens au Burkina Faso (ibid. : 93). Par la suite, sous l'ère coloniale et après les Indépendances, la forme de ces actes migratoires tend à se métamorphoser : imposés par les politiques coloniales, les circuits migratoires se dirigeaient essentiellement vers des régions à fort potentiel économique où une main-d'œuvre conséquente était requise pour assurer la 
« mise en valeur de la terre ». Ils finissent par être plus spontanés et individuels même si le rôle de la communauté familiale et villageoise demeure prégnant.

Néanmoins, quelle que soit la forme migratoire instituée à travers le temps, les référents axiologiques émis par les aventuriers prennent leurs racines dans cette morale consubstantielle à des pratiques initiatiques plus anciennes. L'aventurier se pense en tant que projet social produit à l'aune d'un imaginaire métissé qui dépasse largement le cadre des ethnoscapes ${ }^{19}$ locaux. Dans cette perspective, l'aventure doit être vue en tant que rite contemporain, une recomposition historique à la croisée de laquelle s'enchevêtrent des récits référentiels multipolaires.

\section{À la conquête de l'honneur}

Si la pratique de l'aventure est un rite contemporain qui marque un changement ontologique de l'individu, elle doit être également considérée comme un rite d'appropriation d'un statut social. Parti parfois en quête de numéraire et toujours guidé par la quête de soi-même, l'aventurier ne cesse pas pour autant de se construire par rapport à ceux qu'il a quittés. Famille, communauté villageoise, cercles sociaux de nature diverse sont ces entités identitaires à échelles et influences variables à travers le prisme desquelles l'individu tisse le sens de son existence et s'ancre dans le monde afin d'y imprimer son rôle et sa position sociale. En d'autres termes, l'individu se pense en regard de ce qu'a appelé Alain Marie (1997 : 70) l'habitus communautaire, "structuré par les principes de la solidarité, de la hiérarchie, de l'identité collective et de l'expression corrélative des pulsions individuelles » ou véritable « chez soi » pour l'aventurier, le bayiri ${ }^{20}$ des Moose par opposition à weoogo, la brousse. De surcroît, conséquemment à l'ère coloniale et au vaste mouvement de la mondialisation, la diversification de ces réseaux de socialisation s'est intensifiée laissant à l'individu le soin d'opérer des différenciations dans les rôles qu'il se donne à jouer.

Dès lors, l'acte migratoire est « une aventure personnelle de l'invention de sa propre place » (ibid. : 410) qui ne peut se comprendre qu'en rapport aux institutions collectives d'appartenance de l'aventurier. L'aspect économique est l'un des révélateurs les plus éloquents de ces liens symboliques.

19. Arjun Appadurai (2005: 91) désigne par ethnoscapes les paysages d'identité de groupe.

20. Bayiri ou littéralement « cour (yiri) de mon père $(b a)$ ». L'un des sens généralement donnés est celui de "patrie », mais il caractérise plus globalement le « chez soi », le lieu anthropologique originel. Nous retrouvons cette même relation dans la langue dioula: le faso, "maison (so) de mon père $(f a)$ » s'oppose à tú (la brousse ou la forêt), donc à l'aventure exprimée par l'idiome «tungan ». Ajoutons que dans le langage courant, tungan désigne également la Côte-d'Ivoire («aller au tungan »), destination historique de l'émigration burkinabè. 
En effet, l'importance des transferts de fonds issus des migrations ${ }^{21}$ est le signe du lien quasi ombilical que les aventuriers perpétuent avec leurs réseaux communautaires divers. Loin de chez eux, les aventuriers renforcent leur insertion dans les systèmes d'échanges collectifs en réinjectant une partie du capital épargné, fruit de leur labeur. Ils permettent ainsi de pérenniser l'efficacité des circuits d'entraide dont les fonctions sont d'assurer socialement ses membres et d'amplifier le développement socio-économique des communautés de référence. En outre, la nature de l'investissement n'est pas exclusivement matérielle. En ayant acquis un savoir original sur le monde, ils peuvent être en mesure, une fois de retour, de mettre leurs compétences au service d'une cause commune ${ }^{22}$. Aussi les départs en aventure apparaissentils comme des tentatives stratégiques de maximiser l'intégration de l'individu dans son contexte d'origine, celles-ci étant médiatisées par les dons matériels et immatériels rendus possibles selon le degré de réussite du parcours migratoire. Par conséquent, à travers l'aventure, ce sont l'interprétation de ces rapports sociaux particuliers et le dynamisme des processus d'intégration individuelle qui se revitalisent.

Dans cette optique, la recherche de richesses matérielles et de soi-même est subordonnée à des désirs d'honorabilité et d'estime de soi concrétisés par l'ambition pour l'aventurier de devenir une clé de voûte des solidarités communautaires, une personne «qui compte», un magister qui, par son action, a su se forger un nom. Si la faiblesse du capital matériel et le peu de perspectives de réalisation de soi dessinent la trame contextuelle des départs, le facteur décisif se situe dans un univers hautement plus symbolique, celui de la réputation. C'est dans cette sphère de l'honneur, matrice des conduites humaines, que l'individu façonne son identité et tire en dernier ressort le sens de ses décisions.

Si pour Alain ce sont les soucis économiques qui minent son quotidien, ses aspirations au départ sont conditionnées par la volonté d'être reconnu positivement par son environnement social primaire :

« Je suis né il y a 42 ans à Thanghin-Dassouri, un village proche de Ouagadougou. Je n'avais pas 25 ans quand j'ai eu le projet de rentrer clandestinement en Europe. J'étais à l'aventure en Côte-d'Ivoire où je travaillais dans les champs. Je suis descendu à Sassandra et, avec d'autres aventuriers, j'ai donné 500000 FCFA à un

21. En guise d'exemple, sur la période 1980-2001, le Burkina Faso a enregistré l'entrée de 990105000000 FCFA dont 812100000000 FCFA en provenance de la seule Côte-d'Ivoire (PACERE 2004 : 58-59).

22. À partir d'une ethnographie conduite dans le village de Kulkinka (Burkina Faso) et sa région, Pierre-Joseph LAURENT (1998 : 204-205) rapporte que la notion de « développement» telle qu'elle est expérimentée localement a été importée par d'anciens jeunes migrants de Côte-d'Ivoire, réunis dans une association au réseau tentaculaire appelée Wend-Yam. L'auteur souligne que la notion de développement renvoie « avant tout à un savoir-faire possédé en exclusivité. [...] Le développement n'est pas une abstraction mais renvoie à des pratiques concrètes. Le développement constitue une expérience initiatique vécue loin du village. Seuls ceux qui possèdent cette expérience sont en mesure de la promouvoir » (ibid.). 
passeur dans l'espoir de monter dans un bateau de marchandises qui devait rejoindre l'Europe. Il nous a trahis et s'est enfui avec nos économies en Guinée. Je suis resté en Côte-d'Ivoire pour travailler, mais j'étais découragé alors je suis rentré au village en 1996.

Je suis allé ensuite à Ouagadougou où j'ai travaillé chez un mécanicien. J'ai sympathisé avec un client qui était enseignant à l'université. Il m'a trouvé un poste de vigile et je suis sur le campus depuis huit ans maintenant. Je travaille aussi sur des chantiers, je ne refuse jamais le travail. Je me suis marié et j'ai un enfant que j'ai inscrit à l'école.

Je suis prêt à repartir, à tenter ma chance pour l'Europe. J'ai un ami qui est récemment parti, malheureusement je n'avais pas les moyens de l'accompagner. Je vise la France, j'aimerais y travailler pendant quatre ou cinq ans et puis revenir chez moi avec mes économies. Je sais que le voyage est dangereux mais je n'ai pas peur de mourir. Soit j'y reste, soit je réussis : c'est "la patrie ou la mort". Si tu prends le risque et que tu meurs, c'est mieux que de ne pas le prendre. Si tu as la chance avec toi, tu t'en sors et puis tu as sauvé ta famille et tes enfants.

Aujourd'hui, je suis là, je ne sais pas comment faire pour m'en sortir. Ici, en Afrique, chaque matin a son problème. Ma femme me demande comment on va faire. L'homme doit amener "l'argent de la popote" mais quand tu rentres à la maison, tu n'as rien... Tous les jours ce sont des maux de tête, toutes les nuits ce sont des insomnies. Au village, si on a un problème, personne ne vient me consulter. Pourtant je suis un vieux, un aîné [...] S'il y a des funérailles ou un mariage, les jeunes viennent collecter. Ils ne passent pas par chez moi car ils savent que je n'ai pas 10000 FCFA à leur donner. Si c'est pour un conseil de famille, je ne suis même pas classé. Pourquoi m'appellerait-on ? Juste pour aider à creuser une tombe [...] Je connais quelqu'un à Ouagadougou, il est rentré en France par les bateaux. On a beaucoup parlé ensemble, parfois il a besoin de moi pour des petits travaux. Il a vécu quatre ans en fraude là-bas. Il a nettoyé les rues, il a gardé des enfants, il a travaillé à l'usine, de jour comme de nuit. Il est revenu au pays et il a créé une entreprise de construction. Les engins et les camions lui appartiennent et il a gagné beaucoup de marchés. C'est devenu un grand patron! Il a beaucoup souffert, mais aujourd'hui il est avec sa famille, alors ça va. Lui, il est vraiment devenu quelqu'un, alors quand on le regarde, ça nous donne aussi le courage de partir tenter notre chance. Vous savez, nous, les Moose, nous n'aimons pas être négligés » (Alain, 42 ans, Moaga, Ouagadougou, février 2008).

Dans une société où le notable n'est plus exclusivement l'héritier d'une autorité traditionnelle, la capacité de l'acteur à donner de son capital et de son être configure la hiérarchie des statuts au sein du groupe. Le magister répond à la représentation valorisée du bienfaiteur, celui qui sauve sa famille, dont les propos ont un écho social et qui est remarqué positivement par son activité. À l'opposé, les facultés restreintes du minister ${ }^{23}$ à participer à la vie de sa communauté de référence nourrissent l'angoisse permanente de l'ostracisme, avatar de la honte, d'une mort sociale qui gâte le nom. Sa faible influence le confine dans l'obscurité des rapports sociaux où il demeure invisible, il est celui que le respect fuit, qui n'est pas classé. De

23. Dans les conclusions de son «Essai sur le don», M. MAuss (2004 : 269-270) nous enseigne que «donner, c'est manifester sa supériorité, être plus, plus haut, magister ; accepter sans rendre ou sans rendre plus, c'est se subordonner, devenir client et serviteur, devenir petit, choir plus bas (minister)». 
surcroît, la réussite des migrants de retour, les «venants » (Schmitz 2008 : 8-9), renvoie sans cesse aux « sédentaires », à travers un jeu de miroirs, le reflet de leur position fragile au sein du tissu social, leur incapacité à être percepi, cet être-perçu dont l'action positive et les qualités morales chevillent le prestige. La réussite de certains aventuriers et les conséquences bénéfiques visibles qu'ont leurs conduites altruistes au sein de la société offre des scénarii qui proposent des voies d'accès efficaces vers une mobilité sociale ascendante. Grâce à son projet, l'aventurier espère quitter le seuil de ce statut de dépendant pour devenir ce personnage de haut rang, ce dignitaire que l'on observe, que l'on écoute et qui, par sa capacité de donner, s'est créé un réseau d'obligés, témoin de son influence. Les échanges apparaissent dans leur symbolisme comme des mécanismes distributeurs de pouvoir et des transactions d'honneur qui définissent la place de chacun, les efforts financiers et les investissements consentis par les migrants étant en quelque sorte ce qu'a nommé Marcel Mauss (2004 : 220) « la monnaie de la renommée ».

"Faire quelque chose pour ma famille, c'est mon ziiri [honneur]. » Aventurier moaga

Chez les Moose, l'homme d'honneur (ziirsoba, «le propriétaire de l'honneur ») est celui qui se rend utile pour la société, celui qui transmet, c'està-dire qui donne, qui partage et qui détient le pouvoir de rendre. L'honneur est donc mesurable à l'écot qu'apporte l'acteur à son groupe, ce dernier remboursant sa dette par le don de capital symbolique, à savoir de reconnaissance, de réputation, de prestige. L'individu interagit dans un climat où prévaut le jugement positif et la valorisation sociale, où la honte (yande) entraîne l'exclusion sociale car "[l'homme inutile] sera considéré comme hors société, c'est-à-dire yalma» (Laurent 1998 : 188). Rien n'est pire que la honte et, dès l'enfance, la communauté imprime chez l'individu les codes moraux nécessaires à son intégration dans le tissu social. Aussi, l'estime qu'un individu témoigne à quelqu'un procède-t-elle des référents culturels qui modèlent des systèmes axiologiques de l'acceptable à la source desquels l'individu va adopter les conduites qu'il juge préférables. Dès lors, si l'on considère que l'individu n'est jamais aussi vulnérable que lorsqu'il est attaqué dans ce qu'il a de plus sacré, c'est-à-dire son identité, sa face ou simplement son honneur, il n'est plus surprenant que l'aventurier choisisse de tenter sa chance ailleurs, malgré les périls, que « de souffrir devant ses parents, ses amis et ses ennemis » (aspirant aventurier moaga) car, tel l'adage, ne vaut-il pas mieux «mourir à l'étranger qu'humilié chez soi » ${ }^{24}$ ? L'aventure est en ce sens un acte salutaire, une catharsis morale, un véritable baroud d'honneur.

24. Parole recueillie à Ouagadougou auprès d'aventuriers en devenir. 
Enfin, en partant, le migrant endosse l'identité valorisée de l'« hommecourage » auprès des siens. Il devient celui qui cherche à assumer son rôle social, celui qui affronte son destin pour le bienfait de sa communauté, qui fait montre d'intentions louables. Afficher ces ambitions-là, c'est déjà montrer que l'on est un homme d'honneur, un homme prêt à quitter le monde sensible la tête haute en ayant affronté de face ses difficultés et les périls de la honte : «La mort vaut mieux que la honte » (Tiendrébéogo 1963: 60) pour les Moose ; « la patrie ou la mort ${ }^{25}$ » devisent les Burkinabè ; « la mort mais pas la honte ${ }^{26} »$ pour les Sénégalais. Toutefois, comme nous l'avons déjà évoqué, plonger dans l'aventure implique une mise sous silence volontaire de son existence sociale. "Qui va chercher fortune doit laisser sa dignité sur le seuil de sa case » préviennent les Peulh de Guinée (Amselle 1976 : 91). S'engager sur la route revient à confier son honneur et son nom à sa communauté qui sera en charge de faire vivre le souvenir de celui qui s'absente pour un temps, peut-être pour toujours. L'anonymat relatif au déracinement agit comme un succédané identitaire protecteur dont le but est de préserver l'intériorité de l'individu. Les humiliations infligées à l'aventurier sont par nature dérisoires en regard de l'importance qu'il accorde à son image et à sa place dans sa société originelle, s'étant dépouillé de ce qui fonde son existence (son nom), les souffrances ont moins de prise sur son être le plus profond. Même si l'aventurier continue à souffrir dans son amour-propre, il supporte les épreuves car «la souffrance qui vient des étrangers fait moins mal que celle infligée par les siens » (Dacher 2003 : 159).

Par conséquent, tout se passe comme si l'aventurier, pour devenir honorable, devait pour un temps abandonner toute prétention à l'estime de soi et subir la dépréciation de lui-même; comme si pour devenir visible, il devait se confiner à l'invisibilité le temps de son absence ; comme si pour sortir de la souffrance, il devait intensifier son martyre; comme si pour obtenir une place qu'il juge convenable dans le monde, il devait la perdre temporairement ; comme si pour devenir immobile, apanage des gens prestigieux $^{27}$, il était obligé de se mouvoir dans l'inconnu.

25. «La patrie ou la mort » est une version abrégée de la devise nationale burkinabè héritée de la révolution sankariste : «La patrie ou la mort, nous vaincrons. »

26. Expression qui clôt l'hymne national sénégalais : « Mais que si l'ennemi incendie nos frontières / Nous soyons tous dressés les armes au poing : / Un peuple dans sa foi défiant tous les malheurs, / Les jeunes et les vieux, les hommes et les femmes. / La mort, oui ! Nous disons la mort, mais pas la honte » (DANIEL 2008 : 248).

27. Les principes qui régissent l'acte social de la visite mettent en lumière l'opposition entre la mobilité et l'immobilité qui structure en filigrane les rapports de genre et d'âge. L'immobilité apparaît comme le propre des prestigieux et des aînés, alors que la mobilité caractérise l'infériorité de statut (VINEL 2005 : 206). 
L'aventure formule cette appétence de l'aventurier à être au monde, il s'agit en fait pour lui davantage d'exister au sein des circuits du don qui caractérisent sa communauté de vie que de simplement posséder des ressources matérielles. L'identité de chacun se saisit dans le regard de l'autre et se concrétise dans la position sociale que l'individu occupe dans son univers de vie. Dès lors, il apparaît plus pertinent d'interroger les motivations qui conduisent au départ à l'aune des capabilités des acteurs à interagir positivement dans leurs contextes sociaux, c'est-à-dire par rapport à la liberté réelle dont ils jouissent de choisir la vie qu'ils ont raison de valoriser (Sen 1992 : 122). De ce fait, raisonner ainsi provoque une distanciation interprétative vis-à-vis de la conception matérialiste unilatérale qui perçoit les phénomènes migratoires ouest-africains comme des fuites de situations économiques inextricables.

L'aventure se comprend en réalité à travers un triptyque de motivations dont les deux premiers termes sont subsumés sous le troisième. D'une part, l'aventurier part en quête de biens matériels en tentant de rallier des territoires où il espère pouvoir thésauriser des richesses difficilement accessibles chez lui. D'autre part, l'aventurier est en quête de biens culturels inédits, en témoignent les connaissances qu'il accumule au cours de sa transhumance. Toutefois, ces deux quêtes prennent véritablement leur signification à partir du moment où elles sont connectées au désir affirmé de l'aventurier de se fabriquer, grâce à son action, une place sociale qu'il juge digne, signe de sa solide intégration au sein de sa société. L'ethnographie permet en effet de mettre en lumière des discours s'orientant, implicitement ou explicitement, vers cette volonté, émise par l'aventurier, de s'extraire d'un précariat existentiel afin d'accéder à un nouveau statut social qui, valorisé à ses yeux, le sera également aux yeux des autres. Autrement dit, les logiques opératoires produites par les aventuriers énoncent une quête d'intégration sociale ascendante qui s'affiche en tant que conquête de leur honneur.

L'honneur est catégorie fondamentale de la vie sociale, c'est lui qui détermine la place qu'occupe l'individu, la perception qu'il a de sa propre personne (l'estime de soi) et, dimension cardinale, la perception que les autres acteurs sociaux ont de lui. L'honneur est une force sociale et culturelle prégnante qui régit les conduites des individus, il collabore à la construction des identités individuelles et collectives, il s'épanouit dans l'interaction entre les acteurs, il est au centre de cette mutualité. De surcroît, «il aménage une connexion entre les idéaux en vigueur dans une société et la reproduction que fait de ceux-ci l'individu qui aspire à les incarner » (Pitt-Rivers 1983 : 18). L'honneur est alors systématiquement lié à l'imaginaire axiologique construit par le groupe qui, à travers le prisme des valeurs morales érigées en normes sociales, définit ce qu'il représente. En d'autres termes, l'honneur constitue un modèle de conduites sociales culturellement valorisées auquel les membres du groupe sont encouragés à se référer : si 
telle conduite est honorable, c'est qu'elle correspond à l'idée de perfection que prônent les ethnoscapes où se produit l'action. Quand la honte, valeur sociale négative, participe à l'anéantissement de l'intériorité de l'être, l'honneur exprime au contraire l'idéal que doit poursuivre l'individu, il est le principe par lequel l'acteur peut espérer bâtir sa place dans ses mondes sociaux.

Avant d'être un agent économique, l'aventurier est homo honorabilis, un homme d'honneur en quête d'existence. À travers ses délibérations personnelles, il émet le désir de poursuivre un idéal conforme aux valeurs morales distribuées par ses univers sociaux de référence, et ce quel qu'en soit le prix à payer. Si sortir est effectivement perçu par les aventuriers comme une opportunité d'amélioration des conditions de vie individuelles et communautaires - discours dominant inspiré de l'économie classique — ce qui leur importe c'est avant tout d'obtenir la seule véritable richesse qui compte socialement : un rôle et une place dans le jeu social. En empruntant le chemin, l'aventurier cherche donc à tendre vers une perfection relative aux idéaux édictés par ses groupes, il aimerait devenir ce héros qui, par son courage, a su prouver sa valeur et se rendre indispensable auprès des siens.

À partir de là, l'aventure apparaît comme l'expérience pratique d'une idéalisation opératoire conçue à partir de configurations singulières de la morale en vigueur dans les sociétés de départ. Loin d'être une fuite incarnée par la lâcheté, l'aventure résulte d'un combat où les valeurs de courage et d'abnégation sont centrales, et vise à l'affirmation par l'aventurier de sa présence $^{28}$ au sein de ses communautés de référence. Ainsi, l'aventure est d'abord un ethos qui puise son sens dans le symbolisme inhérent à toute production humaine et qui se comprend comme une stratégie existentielle dont les fondements s'inspirent d'une morale établie, d'une part, par les cultures locales, et, d'autre part par tous les récits rendus accessibles par la mondialisation.

Département d'ethnologie, Université de Strasbourg, UMR 7236.

28. Le terme «présence » utilisé ici ne sous-entend pas nécessairement une présence physique mais forcément symbolique. Autrement dit, l'individu loin de chez lui, par les dons qu'il consent à sa communauté, renforce les liens qui l'unissent à celle-ci, son nom continue de vivre dans les esprits, son souvenir continue d'être perpétué. 


\section{BIBLIOGRAPHIE}

AGIER, M.

2002 Aux bords du monde, les réfugiés, Paris, Flammarion.

Alioua, M.

2008 «La migration transnationale, logique individuelle dans l'espace national : l'exemple des transmigrants subsahariens à l'épreuve de l'externalisation de la gestion des flux migratoires au Maroc», Information sur les Sciences Sociales, $47: 697-714$.

AmSELLE, J.-L. (DIR.)

1976 Les migrations africaines : réseaux et processus migratoires, Paris, François Maspero.

Appadurai, A.

2005 Après le colonialisme: les conséquences culturelles de la mondialisation, Paris, Payot \& Rivages.

BELMONT, N.

1981 «Arnold Van Gennep », in J. HAINARD \& R. KAEHR (dir.), Naître, vivre et mourir : actualité de Van Gennep : essais sur les rites de passage, Neuchâtel, Musée d'ethnographie : 17-31.

BONI, T.

2008 «L'Afrique des clandestins », Information sur les Sciences Sociales, 47 : 681-697.

BRACHET, J.

2009 «Des migrants en transit: sociabilités et territorialités dans le Sahara nigérien », in G. CORTÈs \& L. FARET (dir.), Les circulations transnationales : Lire les turbulences migratoires contemporaines, Paris, Armand Colin : 109-123.

BREDELOUP, S.

1994 «L'aventure contemporaine des diamantaires sénégalais », Politique Africaine, $56: 77-93$.

BRUneau, M.

2009 «Pour une approche de la territorialité dans la migration internationale : les notions de diaspora et de communauté transnationale », in G. CORTÈs \& L. FARET (dir.), op. cit. : 29-42.

Calame, C. \& Kilani, M. (DiR.)

1999 La fabrication de l'être humain dans les cultures et en anthropologie, Lausanne, Payot Lausanne.

Conombo, J. I.

1989 M'Ba Tinga : traditions des Mossé dans l'empire du Moogho-Naba, Paris, L'Harmattan. 
Cortès, G. \& FARET, L. (DIR.)

2009 Les circulations transnationales : Lire les turbulences migratoires contemporaines, Paris, Armand Colin («Collection U, Sciences humaines et sociales »).

DACHER, M.

2003 «Chroniques des violences ordinaires, parcours d'un jeune paysan burkinabé immigré en Côte-d'Ivoire », Journal des africanistes, 73 (2) : 137-161.

DANIEL, S.

2008 Les routes clandestines: l'Afrique des immigrés et des passeurs, Paris, Hachette Littératures.

ESCOFFIER, C.

2008 Transmigrant-e-s africain-e-s au Maghreb : une question de vie ou de mort, Paris, L'Harmattan.

Fiéloux, M. \& Kambou, B.

1993 Biwanté : récit autobiographique d'un Lobi du Burkina-Faso, Paris, Karthala.

GatTi, F.

2008 Bilal: sur la route des clandestins, Paris, Liana Levi.

Goguel D'Allondans, T.

2002 Rites de passage, rites d'initiation: lecture d'Arnold Van Gennep, Québec, Presses de l'Université de Laval.

HiLy, M.-A.

2009 «L'usage de la notion de circulation migratoire », in G. CORTÈs \& L. FARET (dir.), op. cit. : 23-42.

JEFFREY, D.

2005 [2003] Éloge des rituels, Québec, Presses de l'Université de Laval.

KILANI, M.

2009 Anthropologie : Du local au global, Paris, Armand Colin.

LAURENT, P.-J.

1998 Une association de développement en pays mossi : le don comme ruse, Paris, Karthala.

LÉPINe, J. \& VAlluy, J.

2006 Une marche en liberté : émigration subsaharienne. "Jean-Paul DzokouNewo : la traversée d'un enfer européen au Maghreb », Paris, Maisonneuve \& Larose.

MARIE, A. (DIR.)

1997 L'Afrique des individus, Paris, Karthala.

Mauss, M.

2004 [1950] «Essai sur le don», in M. MAuss (dir.) Sociologie et anthropologie, Paris, PUF : 143-279. 
MigReUROP

2006 Guerre aux migrants : le livre noir de Ceuta et Melilla, Paris, Syllepse.

NDiAye, P.

2008 La condition noire : essai sur une minorité française, Paris, Calmann-Lévy.

PACERE, F. T.

2004 Burkina Faso : migrations et droits des travailleurs (1897-2003), Paris, Karthala.

Pian, A.

2009 Aux nouvelles frontières de l'Europe: l'aventure incertaine des Sénégalais au Maroc, Paris, La Dispute.

PITT-RIVERS, J.

1983 Anthropologie de l'honneur : la mésaventure de Sichem, Paris, Le Sycomore.

SAHLINS, M.

1976 Âge de pierre, âge d'abondance : l'économie des sociétés primitives, Paris, Gallimard.

SARR, F.

2008 «Crise de la citoyenneté en Afrique et responsabilité des élites : la question de la migration clandestine », Information sur les Sciences Sociales, 47 : 715-730.

SCHAEFFER, F.

2009 «La circulation migratoire, révélatrice de la structuration sociospatiale du champ migratoire marocain », in G. CORTÈs \& L. FARET (dir.), op. cit. : 61-72.

Schmitz, J.

2008 «Migrants ouest-africains vers l'Europe : historicité et espaces moraux », Politique Africaine, 109 : 5-15.

SEN, A.

1992 Repenser l'inégalité, Paris, Éditions du Seuil.

Simmel, G.

2002 La Philosophie de l'aventure, Paris, L'Arche.

TIENDRÉBÉOGO, Y.

1963 «Histoire traditionnelle des Mossi de Ouagadougou», Journal des Africanistes, 33 (2) : 7-44.

TimerA, M.

2009 «Aventurier ou orphelins de la migration internationale ? Nouveaux et anciens migrants "Subsahariens" au Maroc », Politique Africaine, 115 : 175-195. 
VINEL, V.

2005 Des femmes et des lignages: ethnologie des relations féminines au Burkina Faso (Moose, Sikoomse), Paris, L'Harmattan.

\section{RÉSUMÉ}

Cet article présente une introduction à une anthropologie de la mobilité en Afrique de I'Ouest. II s'agit d'esquisser les contours sémantiques d'une pratique communément appelée "aventure » par ses acteurs. L'analyse produite à partir de données ethnographiques recueillies au Burkina Faso entre 2008 et 2010 permet de relativiser l'hypothèse de la pauvreté matérielle souvent évoquée comme étant la motivation essentielle qui pousse les aventuriers à emprunter le chemin de l' « extérieur ». La réalisation de soi, l'émancipation individuelle de situations sociales jugées parfois trop contraignantes, la soif de découverte ou de nouveaux mondes et, fondamentalement, la recherche de l'honneur concourent à la forte mobilité des populations ouestafricaines. L'aventure, souvent conçue comme un processus initiatique, se construit à bien des égards autour de référents axiologiques valorisés par les contextes locaux.

\section{ABSTRACT}

Ethnological Approach of Sub-saharan Irregular Migrations: The Adventure, or from the Ontogenesis to the Conquest of Honour. - This article features an introduction to an anthropology of mobility in West Africa. It sketches out the semantic outlines of a practice commonly referred to as "adventure" by its actors. The analysis is based on fieldworks carried out in Burkina Faso between 2008 and 2010 and helps to put into perspective the widespread assumption which considers material poverty as being the main factor of these out-migrations. Self-realization, individual emancipation from constraining social situations, the discovery of new worlds and, basically, the quest of honour participate in West African populations' strong mobility. The adventure, often understood as an initiatory process, builds up its meaning over axiological referents, enhanced by the local contexts.

Mots-clés/Keywords : Afrique de l'Ouest, Burkina Faso, aventure, honneur, initiation, migrations/West Africa, Burkina Faso, adventure, honour, initiation, migrations. 